to $106^{\circ}$ or $107^{\circ}$ are communicated to their constituent molecules, disorganisation follows. Hence it is intelligible that, if a consumptive patient whose temperature at sea level is $98.4^{\circ}$ he raised to an elevation of $6000 \mathrm{ft}$., such alteration of molecular motion will take place in the blood as to be incompatible with the healthy existence and effective reproduction of the tubercular microbe ; in fact, the patient will be placed in the position of the bird to the splenic fever poison. If this reasoning be sound, wide is the vista with regard to the epidemic levels of yellow fever, malaria, cholera, \&c., which it must open up, and strange the possibilities which might result from the artificial production of the required conditions.

Military Prison, Dublin.

\section{ONE HUNDRED CONSECUTIVE CASES OF ABDOMINAL SECTION.}

By GEO. GRANVILLE BANTOCK, M.D., F.R.C.S. ED., SURGEON TO THE SAMARITAN FREE HOSPITAI.

(Concluded from page E21.)

Hydatids of the peritoneum.-The patient was a young single woman, aged twenty-five, by occupation a cook. When admitted in May, 1886, she gave the following history. She had been aware of "a lump in the stomach" for six or seven years. Three years and a half ago she had retention of urine, which was relieved by passing the catheter two or three times. She then entered a suburban hospital, where she was told she had a tumour. Nine months ago she had a child, and since that time the increase in the size of the tumour had been very marked. On opening the peritoneum, it was at once evident that I had to do with a case of hydatids-which I may say was suspected. The incision was enlarged to about $4 \mathrm{in}$. to admit of extraction of the different tumours before separating them from their connexions with the omentum, \&c. I began by breaking down the connexions of the largest cyst, but they were so firm and the bleeding so great that I was obliged to resort to the method of ligaturing first and then cutting. In all, I removed about half a dozen cysts of various sizes, from that of a hen's egg to that of a small cocoa-nut. One of medium size occupied the right half of Douglas's pouch, where it hung pendulous with a slender attachment to a tag of omentum, where it was felt before operation, and regarded as an enlarged ovary. Down in the left iliac region, one, as large as an orange, lay amongst the coils of intestine. On seizing the exposed portion of the cyst wall between the finger and thumb, it at once gave way, and many daughter cyste, from the size of a pea to that of a walnut, escaped amongst the intestines and were with difficulty recovered. That one eluded my search was evident from the fact that at a subsequent period a small collapsed sac came away through the tube-hole. The cyst wall was so friable that it could only be detached from the intestines by means of the sponge. A small cyst was attached to the edge of the liver, and there was a larger one on the under surface of the liver, very far back. As it would have been impossible to drain these if opened, I let them alone. After thoroughly washing out the peritoneum a drainagetube was inserted, and the patient made a good recovery. She is now in excellent health and able to follow heroccupation. Nor is there any increase in the liver cysts. The weight of the cysts exceeded $2 \frac{1}{1 b}$.

Retro-peritoneal cyst. - This tumour occurred in the case of a young lady aged fifteen, who came under my notice about the end of January last, having been sent to me by Dr. Robson Roose. The tumour had been observed for about eighteen months, and when first discovered was about the size of a Mandarin orange. During this time the patient was subject to attacks of severe pain, often accompanied with fever, the temperature sometimes running up to $105^{\circ}$. There was much bewildering consultation over the unfortunate patient, and the case forcibly illustrates the remarks made by Mr. Lawson Tait in a paper read before the Medical Society of London on Nov. 8th last, when he denounced many consultations with physicians over surgical cases as farcical proceedings. At last, however, Sir Joseph Lister agreed with me as to the propriety of surgical interference, on the ground that there was distinct evidence that the tumour was growing rapidly. Accordingly it was removed on Feb. 16th, 1886, the incision along the outer border of the left rectus muscle extending about an equal distance above and below the umbilical level. The tumour was covered by the mesentery, whose vessels were enormously enlarged, and it lay just over and in close contiguity to the left kidney, for which it was mistaken, owing to theimpulse communicated to that organ on bimanual palpation. and it pushed the splenic flexure of the colon towards the umbilicus. Many ligatures were required in the course of the enucleation, the vessels being doubly secured before division: and, finally, the greater part of the sac was cut away after the application of a double stout ligature. A drainagetube was inserted, as there was still some oozing from the remaining torn surfaces. The patient made a good though tedious recovery, owing to the occurrence of some superficial suppuration in the track of the drainggetube. The temperature only once reached $100.8^{\circ}$, and was above $100^{\circ}$ for only one day. The patient is now quite well. The tumour weighed over $1 \mathrm{lb}$. It is now in the museum of the Royal College of Surgeons. Mr. Eve kindly furnished me with the following report:-" The tumour is composed of a number of inter-communicating loculi, separated by numerous septa, and contained a glairy mucoid fluid. I am quite at a loss to explain its origin, the situation being so unusual, and only conjecture that it originated from some fœtal structure, possibly some of the rudiments of the permanent kidney; it was too high for the Wolffian body. The other possibilities that occur to me are that it was an ovarian cyst which had become separated by twisting of its pedicle, or that it was of the same nature as the cysts which have been found in the great omentum." I talke Mr. Eve's first idea as probably the correct explanation of the origin of this remarkable tumour. It was situated too much behind the mesentery for an ovarian tumour which had become separated from its natural attachments, and its relations in no respect resembled an instance of that kind which occurred in my practice in October, 1884, in which I removed a small ovarian tumour from the right side of the abdomen, and whose pedicle had been severed from the left side.

Incision and drainage.-A married woman, aged thirtyseven, the mother of three children, the youngest of whom was eight years old, was sent to me by Dr. Amand Routh. There was a history of acute disease within the peritoneum in January last, with uncontrollable sickness for two days, severe pain for five days, and fever. At the end of February she was tapped of over half a gallon of a thickish, paleyellowish fluid. In another month she was again tapped of three pints of a thicker fluid of the same appearance, and was recommended to apply poultices orer the abdomen. In a few days the puncture hole began to discharge a thick puriform fluid. After three months' confinement to her bed she began to get about, and at the end of May came under the care of Dr. Routh in the out-patient department of the Samaritan Hospital, but I was unable to admit her until the end of July. She was then in a very emaciated condition; the puncture hole was still open, but only occasionally discharging. An ordinary surgical probe was passed in to its full length, but nothing came out on its withdrawal. I therefore thought I bad to deal with a multilocular tumour suppurating in its centre loculus, and I was not a little surprised in the operation that when the parietes were divided 1 opened direct into a single cyst filled with a purulent-looking fluid, to the amount of about four pints. After washing out the cavity till quite clean with simple warm water, the cyst wall was seen to be lining the whole pelvic cavity, the intestines having been pushed out and the uterus pressed forwards and downwards; in fact, looking into the pelvis was like looking into a hat. It was therefore evident that this was not a case for further interference, and I accordingly closed the wound and left in a drainagetube, and for the purpose of excluding air I filled up the cavity with warm water. After forty-eight hours, when there was a slight odour from the discharge, the cyst was washed out with sulphurous acid several times daily, and the odour at once disappeared. Whilst the temperature previously to the operation daily rose towards evening to $102^{\circ}$, it remained perfectly normal afterwards. In fifteen days she went to a convalescent institution, and in a few weeks more returned home. She presented herself at the hospital in November in perfect health.

Exploratory operations.-The list of seven includes nearly as many different conditions. In the first case the whole 
peritoneum was studded with sago-like granulations, and the patient was much benefited by the removal of the fluid. When last seen, three months after operation, the fluid had not reaccumulated. The diagnosis made was "probably malignant disease." Whether this was correct, or whether the disease may turn out to be of a non-malignant nature, remains to be seen. It is, however, a singular fact that whilst tapping in these cases is always followed by a rapid reaccumulation of the fluid, the operation of abdominal section has been followed, in my experience, by complete immunity, even in cases that have proved fatal in a few months, from the progress of the initial disease.

The second and third were instances of supposed tubal disease: one of the patients being a young married woman, aged twenty-five, with two children, the youngest being six months old; and the other aged forty-six, with one child aged twenty-five. In both cases the adhesions of omentum, and especially of intestine, over the pelvic contents were very extensive, and so firm that there seemed no possibility of reaching the pelvis without rupturing the intestines, and after stopping the oozing from the ruptured adhesions by means of ligatures and the pressure of sponges the wound was in each closed. I have seen the latter of these cases quite recently, and while at the time of the operation, in November, 1885, the uterus was quite fixed in the pelvis, with considerable surrounding tumefaction, it is now quite mobile and the swelling has almost disappeared. This result would seem to disarm criticism directed against the noncompletion of the operation, especially as the patient is already so advanced in years, and yet the attempt appears to me to have been justified by the physical signs and symptoms existing at that time.

The fourth case was one of pupilloma extensively involving the left broad ligament, and the operation confirmed the diagnosis. The patient was a married but sterile lady aged fifty-six. She had been tapped three weeks previously, and the fluid had rapidly re-formed. Abdominal section was performed with the view of taking advantage of the curious fact to which I have just referred, and in this I was not disappointed; for while the patient was under my observation-three weeks-there was no reaccumulation of fluid How long that might have been the case cannot now be determined, as the patient died a few weeks afterwards of an acute pulmonary disease.

In view of Mr. Doran's paper, recently read before the Obstetrical Society of London, on "Papilloma of the Fallopian Tubes," I may here state that I am inclined to believe that many, perhaps the majority of cases in which we find this form of disease, are instances in which the papilloma, taking its origin within, has burst through the tube and then spread itself over the broad ligament and adjacent structures. The last case I shall refer to (the second in the paper above noticed) appears to support this view, and if it be correct, then papilloma of the tube is not so uncommon as Mr. Doran's researches would lead us to suppose.

The fifth case is one of very great interest. On Dec. 8th, 1885, I was asked by Mr. Tapson of Clapham to see Mrs. R aged thirty-eight, the mother of two children, of whom the youngest was seven and a half years old. The history was as follows:-For the last year she had been "generally out of sorts": menstruation, previously normal, had become very irregular, with intervals of two and three months, and the last period occurred in the end of August. By the end of September she began to complain of pain in the right groin, which had gradually become very severe. She therefore consulted Mr. Tapson in November, and he, finding a small tumour in the right ovarian region, requested me to see her. On examination, a tumour, the size of a large orange, was found on the right side of, and closely attached to, the uterus, tender on palpation, non-fluctuating, but elastic. The fundus uteri could be distinctly defined. There was nothing characteristic in the condition of the cervix. As the result of this examination, I gave it as my opinion that the disease was either an enlarged ovary very closely attached to the uterus, or an affection of the (right) Fallopian tuhe, and I advised that Mr. Tapson should watch the case. By the beginning of January the pain had become so severe, coincident with an enlargement of the tumour, that he requested me to take her into the hospital as soon as possible, and she was admitted on January 11th. I was at once struck with the great change that had taken place in the local condition, and came to the conclusion that she was pregnant. Remembering the fact that on my first examination the fundus uteri could be distinctly made out, and recognising the change which had taken place in the cervix,
I further concluded that the pregnancy was Fallopian, and I communicated to this effect with Mr. Tapson, who visited her with $m e$ in the hospital. The sound was passed and entered over four inches, running up on the left side of the mass, where its point could be distinctly felt. The tumour now extended as high as the umbilicus, and lay almost wholly on the right of the median line. She was seen and examined by several of my colleagues, the majority of whom agreed with meas to the existence of pregnancy. The pain continuing as severe as ever, and no signs of uterine disturbance having resulted from the use of the sound, it was determined. with the concurrence of Mr. Tapson and my colleagues, that abdominal section should be performed to avoid the risk of rupture, which appeared very probable from the severity of the pain. The case was thoroughly explained to the patient and her husband, and by the former the risk was most cheerfully accepted as affording the prospect of relief from her well-nigh intolerable sufferings. I must state that, had I been guided solely by the results of this examination and had not seen the case at an earlier period, I should have looked upon the case as a uterine pregnancy of which the pain could not be explained. After a lapse of eight clear days from the passing of the sound, the patient was submitted to operation. On opening the abdomen it was at once apparent that, after all, the foetus was in the uterus. On the left side the ovary was in its normal position, and there was a considerable space between the uterus and the side of the pelvis. On the right side the ovary was low down behind the tumour, which was in close contact with that side of the pelvis; in fact, the ovary had not risen out of its usual position in the unimpregnated state. Very little of the tube beyond the fimbriæ could be made out. It is almost needless to state that I at once closed the abdomen. The temperature on the following day rose from $99^{\circ}$ to $100.2^{\circ}$ (for four hours), and by the next day was quite normal. On the seventh day uterine action set in, and a fœetus weighing $1 \mathrm{lb}$, with a placenta $6 \frac{1}{2} \mathrm{oz}$., was expelled in a few hours. The patient had an uninterrupted convalescence, got quite rid of the pain, and returned home on Feb. 16th, much gratified with the result. Some there are, probably, who will say this was a simple uterine pregnancy. I think a careful study of the history will not support that contention. There can be no doubt that the uterine cavity did not contain the fœtus in the first instance, for both Mr. Tapson and myself were agreed that the body of the uterus could be distinctly defined at our first examination. The conclusion $I$ have come to is that the ovum was arrested in the uterine extremity of the tube, and gradually worked its way into the uterine cavity. This view is supported by a case reported in THE LANCET of Jan. 16th, 1886, by Dr. Alexander Hay, and by another brought before the Obstetrical Society of London in October, 1885 , by Dr. Griun, in both of which the physical signs very much resembled my case in its early stage. On submitting this report to $\mathrm{Mr}$. Tapson, he replied, under date Dec. 10th: "I have nothing to add except that I saw her (Mrs. R.) about a week since, and she is in better health than she has been for years."

The sixth case was that of a single lady aged twentythree, who till within a year had enjoyed good health. On dividing the peritoneum, which was very much thickened, a small quantity of a clear amber-coloured fluid escaped, and a very thin and delicate membrane presented, within which was contained a whitish flocculent fluid to the amount of two pints. No intestine was seen, but on removing as much as possible of this membrane the exposed surface presented a glistening and injected appearance. The cavity appeared to be limited by adhesion to the parietes. It was washed out with warm water, and the wound completely closed. The disease appeared to be of a tubercular nature. On the evening before operation the temperature was $100.6^{\circ}$ and it rose to the same height on the same evening, but for the next two days was nearly normal; it then began to rise in the evening for about ten days. At the end of three weeks she was out of bed, and when about to return home she complained of hypogastric pain, and on the twentythird day a small quantity of fluid, similar to that removed at the operation, escaped. The temperature then became normal, and she returned home on the thirty-first day with a small sinus. This had not closed when I last heard of the patient, at the end of November, though she was then in very good health.

The seventh and last case was that of a married sterile 
roman, aged fifty-one, whose case had been a puzzle to aore than one, at one time presenting the signs of a cyst nd at another those of ascites. As the latter had become redominant, and no definite explanation could be offered if this condition, I decided to explore rather than tap the ratient. I operated on her on Nov. 10th last, removing ten lints of free fluid. There was no disease of the pelvis or bdomen to account for the fluid. The body of the uterus ppeared to have completely atrophied, and what should lave been the body of that organ formed the floor of the elvis. No ovary could be found. At the splenic flexure ihe colon adhered to the parietes. The temperature renained normal, and when the patient left the hospital on he nineteenth day there was no reaccumulation of fiuid.

Incomplete operation.- A married sterile lady, aged fortyive, consulted me on Sept. 16th, 1885, complaining of a tragging pain in the pelvis, a severe pain in the region of the bladder, frequent micturition, and sometimes retention of urine. On examination, an elastic mass filled the pelvis, bulging down into the vagina, and pressing the uterus upwards and forwards, close up behind the pubic symphysis. The cervix was almost obliterated; the uterine cavity measured two inches and a half, and was directed to the lett side. I recommended operation, though I could not urge it, and the patient preferred to wait for two or three months. She returned at the end of December with all her symptoms much aggravated, especially the bladder trouble; and having made up her mind for active treatment, I operated on Dec. 26th. The pelvic tumour was then distinctly fluctuating. On opening the abdomen, the first thing that presented itself was the right Fallopian tube close to the cornu of the uterus, forming a mass as large as a hen's egg, and having a knuckle of intestine very intimately attached to it. On separating these, the muscular coat of the intestine was laid bare, and the edges of the peritoneum were brought together by several points of continuous suture. In the course of the separation the tube was opened into and seen to contain papilloma. External and posterior to this was a cyst with thin walls, which was aspirated, and yielded about half a pint of a thin fluid like weak tea. An attempt was now made to separate the collapsed cyst, and in doing this another cyst, deeper in the pelvis, was opened, with the escape of a similar fluid. I was, however, obliged to desist from the attempts at separation, and had to be content with freely laying open the cysts and removing a portion of cyst wall together with the papillomatous portion of the tube. I then washed out the cavity with warm water, put in a drainage-tube, and closed the wound. The patient went on very well for forty-eight hours, then the urine became scanty, the pulse rapid, the temperature rose to $102^{\circ}$, and the mind was obscured. With the decreasing urinary secretion, which ultimately ceased, the pulse increased in rapidity, with a falling temperature, the patient became comatose, and died towards the third day. No postmortem examination was obtained. This case, I think, illustrates the danger of delay when there are symptoms of urinary pressure and obstruction. I have already spoken of the papilloma, and it is scarcely necessary to point out the nec-ssarily fatal character of the disease, which must in a very short time have opened into the intestine.

This case, then, constitutes the last of the consecutive series of one hundred cases of abdominal section-consecutive not in point of date, but according to the table. All of them were performed between Feb. 13th, 1885, and Nov. 17th, 1886, inclusive, and without any of the so-called antiseptic precautions; some of the hospital cases were done whilst patients were dying of septicæmia in neighbouring wards, and I did not adopt the precaution of hanging up a wet carbolised sheet over the door. But the greatest care was taken to ensure cleanliness, which I described some years ggo as "the most efficient of all antiseptic systems and the only perfect one." That, I think, is borne out by the results. It would be foreign to my purpose to institute any comparison with the results of other operators. The above narrative of on hundred consecutive cases is a statement of facts at the service of those who have a mind to exercise their ingenuity in the manipulation of statistics.

Granville-place, Portman-square, $w$.

AT a well-attended meeting of the governors and friends of the Charing-cross Hospital on the 9th inst, a committee was appointed to organiso a special appeal on behalf of the funds of the institution.

\section{OASE OF \\ GOITRE, FOLLOWED BY ASPHYXIA; TRACHEOTOMY; DEATH.}

\section{BY J. B. II URRY, M.A., M.D.}

EMILY B-, aged thirteen, when first seen, was suffering from a goitre of very moderate dimensions, which had been growing for a year. No history of goitre in the family. The first symptoms appeared about Nov. 3rd, 1886 when the girl began to complain of dyspncen, stating that it obliged her to rise early in the morning. This continued till Nor. 7th, when Mr. Christian of Reading (lo whom I am indebted for permission to use these notes) was sent for, who saw the girl at 12 noon, and found her with some, but not urgent, dy spnca; the breathing was noisy, but not stridulous; no signs of inflummation or feverishness; she ate well that day. At 11.30 P.M. the same day, Mr. Christian was again sent for, and on arrival found the girl in a state of extreme dyspnœa, and only partly conscious. He immediately came for me to assist him, and we both returned as quickly as possible, provided with the means of performing tracheotomy. On reaching the girl's house at 12.30 A.M. on Nov. $8 \mathrm{th}$, all the signs and symptoms of asphyxia were present: the face was pale; conjunctival reflex almost abolished; complete unconsciousness; breathing excessively laboured. Mr. Christian proceeded at once to open the trachea between the cricoid and the enlarged isthmus of the thyroid gland. No anæsthetic was necessary, owing to the state of unconsciousness. On making the superficial incision, much venous hæmorrhage occurred; as soon as this had for the most part ceased, and the girl evidently being at point of suffocation, the trachea was incised, and a small silver tube rapidly introduced. Air passed in and out through it, but not freely, and a moderate amount of blood came with it at first. A larger tube was therefore substituted, but even then the breathing appeared very obstructed. The tube was kept clear by a feather, but the breathing was far from satisfactory; indeed, the asphyxia became more and more complete, till at about 2 A.M. on Nov. 9 th (i.e. about half an hour after tracheotomy) death occurred.

I made the post-mortem examination on the same day, at 6 P.M., by removing the larynx, œsophagus, and the entire thyroid growth. On dissecting the mass, and opening the larynx and trachea from behind, nothing abnormal was found, except a moderately large goitre and a bilaterally compressed trachea. The incision made by the tracheotomy began immediately below the cricoid, and measured about two-thirds of an inch in lengtb, two rings being cut through. A short distance below the incision, the trachea was considerably flattened from side to side for the distance of an inch; both walls were affected, but the left one most; instead of being convex outwards, it was slightly so inwards There was no softening or kinking of the trachea. On introducing the tracheotomy tube as it would have lain during life, the lower end was found to reach nearly to the lower portion of the region of flattening. The tumour itself was nearly symmetrical on the two sides. The isthmus was continuous with the right lobe, but separated from the left by a groove; it measured one iuch from above downwards and also from before backwards, and began immediately below the lower end of the incision. Each lobe was about as large as two hen's eggs; together they entirely encircled the trachea, and behind it came into contact for about the surface of one inch in an antero-posterior direction, and two inches from above downwards; they separated the oesophagus from the trachea. On making incisions into the thyroid, the tissues were uniformly firm; there were no cysts visible to the naked eye, nor any sof tened portions. On bringing the two lobes into the position they occupied during life, the lumen of the trachea was seen to be reduced to a narrow anteropnsterior slit (the Sübelscheide of the Germans). The relation of the recurrent laryngeals to the growth was unhappily not noted.

Remarks.-This is one of the few recorded instances of sudden death from goitre, and the interesting question arises, What is the cause of death? No perfectly satisfactory answer has been given, especially in regard to the cases where (as in the above) there is no ovldence of sudden increase in size. The view generally adopted is, I believe, 